\title{
Uso de los servicios de urgencias en Bogotá, Colombia: Un análisis desde el Triaje
}

\author{
Use of emergency services in Bogotá, Colombia: An analysis from the triage
}

Fredy Guillermo Rodríguez-Páez ${ }^{*}$ orcid.org/0000-0001-5067-1353

Wilson Giovanni Jiménez-Barbosa' orcid.org/0000-0002-0467-0365

Francisco Palencia-Sánchez² orcid.org/0000-0002-8126-7748

1 Universidad de Bogotá Jorge Tadeo Lozano. Bogotá, Colombia

2 Doctorado Interfacultades en Salud Pública, Universidad Nacional de Colombia. Bogotá, Colombia

\section{Resumen}

Introducción: Los servicios de urgencias son puntos críticos para la calidad de la atención. Comprender los motivos de su uso aporta al mejoramiento de los sistemas de salud. Objetivo: Se describieron las características de afiliados al régimen contributivo que usaron servicios de urgencias en tres hospitales de nivel III de complejidad en Bogotá, así como las impresiones diagnósticas y distribución cronológica de la demanda, de acuerdo con los datos obtenidos al momento de ser clasificados en el Triaje. Materiales y métodos: Se realizó un estudio de corte transversal, con base en registros de Triaje en el lapso comprendido entre 1 de octubre de 2012 a 31 de marzo de 2013. Resultados: Un tercio de los registros fueron clasificados como consultas médicas no urgentes; el día lunes tuvo el mayor número de atenciones; las urgencias tuvieron un comportamiento diurno; variables como: sexo femenino, condición de afiliación como cotizante al sistema de salud y tener edad para trabajar, se asociaron con diferencias en la clasificación del Triaje al momento de demandar las urgencias. Conclusión: El uso de los servicios de urgencias está influenciado por la relación entre: características demográficas, condición de afiliación al sistema de salud y clasificación del Triaje.

Palabras clave: Servicios de salud; triaje; práctica de salud pública; urgencias médicas; mal uso de servicios de salud. (Fuente: DeCS, Bireme).

\begin{abstract}
Introduction: Emergency services are critical points for the quality of care. Understanding the reasons for their use contributes to the improvement of health systems. Objective: To describe the characteristics of members of the contributory regime who used emergency services in three level III hospitals of complexity in Bogotá, as well as the diagnostic impressions and chronological distribution of the demand, according to the data obtained at the time of being classified in the triage. Materials and methods: A cross-sectional study was conducted based on triage records from October 1, 2012 to March 31, 2013. Results: A third of the records were classified as non-urgent medical consultation. Monday had the greatest number of attentions. Emergencies had daytime behavior. Variables such as: female sex, membership status as a contributor to the health system and age to work were associated with differences in the classification of triage at the time of demand for emergencies. Conclusion: The use of the emergency services is influenced by the relation between: demographic characteristics, condition of affiliation to the health system and classification of the triage.
\end{abstract}

Key words: Health services; triage; public health practice; emergencies; health services misuse. (Source: DeCS, Bireme). 


\section{Introducción}

De acuerdo con numerosos estudios, los servicios de urgencias presentan una elevada y creciente demanda por parte de los usuarios, en muchas ocasiones, debido a motivos que no son pertinentes para ser atendidos en ellos. Esto ha conllevado a que los servicios de urgencias cada vez jueguen un papel más relevante en los sistemas de salud a nivel mundial por factores asociados a: las condiciones de oferta de los mismos, las características de quienes los demandan, y a las condiciones de organización de los sistemas de salud de los que hacen parte(13).

Dentro de los factores asociados a la oferta se destacan: el empleo de recursos técnico diagnósticos costosos, el compromiso de talento humano especializado y la destinación de áreas e infraestructuras físicas, así como la localización geoespacial que facilite el acceso de los usuarios. En cuanto a las características de la demanda son relevantes los siguientes: demográficos, geográficos, ambientales, administrativos, económicos, sociales y culturales. Por último, en relación con la organización de los sistemas de salud, se resalta la forma en que el modelo de atención primaria afecta el nivel de demanda de los servicios de urgencias ${ }^{(1,4)}$.

La combinación de los factores anteriormente relacionados, junto con las características y condiciones de organización de los servicios de urgencias particulares de cada Institución Prestadora de Servicios de Salud (IPS) han derivado en problemas tales como: primero, aglomeración y prolongación en los tiempos de espera que pueden ocasionar mala atención a los usuarios; segundo, frustración del personal de salud causada por la saturación excesiva de los servicios de urgencias, la cual además puede derivar en que se produzcan errores y omisiones graves en el proceso de atención; y finalmente, el incremento en el costo de atención a causa del compromiso del talento humano y de recursos tecnológicos altamente especializados para la atención de patologías solucionables en otros servicios menos costosos como los de consulta externa(1,5-7).
En Colombia, el Triaje es la metodología más común para determinar si el paciente puede ser considerado "realmente urgente" Esta metodología clasifica los enfermos que demandan atención en el servicio de urgencia teniendo en cuenta criterios de gravedad clínica con el fin de diferenciar entre aquellos que requieren atención inmediata de quienes no la requieren, logrando así, un uso más eficiente de los recursos del servicio de urgencias ${ }^{(8-10)}$. El Triaje tiene como objetivos: identificar de forma rápida los pacientes con alteraciones en su estado de salud que pueden poner en riesgo su vida; disminuir la congestión en las salas de espera de urgencias; proveer información al paciente y a su familia sobre los tiempos de espera y; establecer orden en el desarrollo del trabajo en el servicio(8,11-13).

Si bien el Triaje desarrollado en Francia es la metodología más difundida para la clasificación del riesgo en urgencias, existen otras menos empleadas, como el Sistema de Clasificación de Manchester (Manchester Triage System), creado en Inglaterra en 1994 y, que ha sido incorporado en los sistemas de salud de países como Brasil, Portugal e Inglaterra(14-16).

El Triaje diferencia los niveles de prioridad asignando colores de los que se deriva un tiempo estimado de espera para recibir la atención médica; así: Rojo, emergencia (inmediata atención); naranja, muy urgente (atención igual o menor a diez minutos); urgente, amarillo (tiempo máximo de atención igual o inferior a sesenta minutos); azul, condiciones estándar (periodo de espera igual o inferior a ciento veinte minutos) y; verde, no urgente (tiempo de espera igual o inferior a doscientos cuarenta minutos). El sistema sugiere que el personal de enfermería debe ser el responsable de adelantar este proceso de clasificación; esta debe ser iniciada dentro de los diez primeros minutos posteriores al arribo del paciente al servicio de urgencias y se realiza mediante la selección de un diagrama de flujo específico (dentro de un total de 55 posibles) para cada paciente, gracias a la aplicación de un grupo de preguntas que discriminan los factores de riesgo que presenta $^{(9,12) \text {. }}$ 
En Colombia el acceso a los servicios de salud incluido el de urgencia se garantiza por medio del Sistema General de Seguridad Social en Salud (SGSSS) el cual cubre la población del país mediante tres regímenes, a saber: Contributivo, Subsidiado y Especiales(17).

En lo referente al contributivo, se afilian los trabajadores formalmente empleados, los pensionados y los trabajadores independientes, quienes se denominan cotizantes y los cuales tienen derecho a incluir a los miembros de su grupo familiar, a quienes se les denomina beneficiarios. Al régimen Subsidiado se afilian los grupos familiares de personas de bajo estrato socioeconómico usualmente en el mercado laboral informal con una reducida o nula capacidad de pago. Finalmente, a los regímenes especiales pertenecen las Fuerzas Militares y de Policía; los educadores públicos de nivel formación básica y media y; los trabajadores de la Empresa Colombiana de Petróleos (ECOPETROL) $^{(17) .}$

Los afiliados a los regímenes contributivo y subsidiado eligen de manera libre la Entidad Prestadora de Salud (EPS) de su predilección, la cual debe garantizar su atención mediante una red de Clínicas y Hospitales, las cuales se denominan IPS. Los regímenes especiales, cuentan con autonomía para brindar los servicios de salud a sus afiliados(17,18). Si bien para el año 2016 el SGSSS en Colombia logra en $94 \%$ de población cubierta ${ }^{(19)}$, por regímenes, al momento de realizar esta investigación, la población afiliada al SGSSS en 2013, a nivel nacional se distribuía así: contributivo 20.150.266 afiliados (46,6\%); subsidiado 22.669 .543 (52,5\%); y exceptuados (Magisterio, Ecopetrol, Fuerzas Militares y Policiales) 387.664 personas $(0,9 \%)$. En Bogotá, para el mismo año, la población afiliada al SGSSS se distribuía de la siguiente manera: contributivo 5.592.369 afiliados (81,1\%); subsidiado $1.267 .841 \quad(18,4 \%) ; \quad y \quad$ exceptuados 35.578 personas $(0,5 \%)^{(20)}$.

Para el año 2011 en las EPS del régimen contributivo se atendieron 7.881.271 urgencias, de las cuales el $50 \%$ se atendieron en la ciudad de Bogotá. Los primeros diagnósticos en población adulta fueron dolor abdominal y pélvico $8 \%$, seguido de infección intestinal aguda $7 \%$, infección respiratoria aguda $6 \%$, cefalea $5 \%$ y dorsopatías con $3 \%{ }^{(21)}$, mientras que para el año 2014, los primeros diagnósticos en las atenciones de urgencias para población adulta del régimen contributivo en Colombia fueron enfermedades del sistema respiratorio, ciertas enfermedades infecciosas y traumatismos(22).

En Colombia, la literatura sobre este tema es escasa y la existente está enfocada a análisis de morbilidad sentida, acceso al servicio, morbilidad de consulta de urgencias y demanda inadecuada del servicio(2,6,11,23,24). Por ende, investigaciones encaminadas a ayudar a comprender estos problemas, aportan elementos que facilitan la toma de decisiones tanto a nivel micro como macro de las organizaciones que conforman los sistemas de salud.

La mayor proporción de afiliados del régimen contributivo en Bogotá en relación con la observada en el país, explicó la decisión de que la investigación se centrara en el análisis de los datos del Triaje realizado en los servicios de urgencia a afiliados a dicho régimen en la ciudad de Bogotá. De esta forma, se planteó como objetivo general: describir las características de los afiliados al régimen contributivo que usan los servicios de urgencias en tres instituciones hospitalarias de nivel III de complejidad en la ciudad de Bogotá D.C., así como las impresiones diagnósticas y la distribución cronológica de la demanda, de acuerdo con los datos obtenidos al momento de ellos ser clasificados en el Triaje.

\section{Materiales y métodos}

Estudio de corte transversal, en el cual se realizó un análisis, con base en información comprendida en el lapso entre 1 de octubre de 2012 a 31 de marzo de 2013 (la decisión de cubrir este período de tiempo se debió al requerimiento establecido por los financiadores de la investigación), proveniente de tres IPS de tercer nivel de complejidad, localizadas en la ciudad de Bogotá D.C., que cumplieron con los siguientes criterios de selección: ser de 
naturaleza privada; atender población afiliada al régimen contributivo; que por su ubicación geográfica facilitaran el acceso a los usuarios desde diversos puntos cardinales de la ciudad (una en zona centro oriente de la ciudad, otra en la zona sur occidente y la tercera en la zona noroccidental); que se comprometieran a enviar los Registros de Triaje (RT) de su servicio de urgencias correspondientes al período de tiempo definido y que fueran afiliadas a la Cámara Sectorial de Salud de la Asociación Nacional de Industriales (ANDI) ${ }^{(25) .}$

Subsecuentemente, se recibieron los RT solicitados a las IPS, los cuales contenían las siguientes variables:

1. Tipo de afiliación del paciente atendido: cotizante o beneficiario.

2. Fecha y hora de atención: de acuerdo con el huso horario utilizado en Colombia. Para facilidad de los análisis, se agruparon los horarios de atención en segmentos del día de acuerdo con la siguiente división: madrugada, desde las cero horas hasta las seis horas del día; mañana, a partir de las seis horas, hasta las doce horas del día; tarde, desde las doce horas hasta las dieciocho horas del día y; noche, a partir de las dieciocho horas, hasta las veinticuatro horas del día.

3. Sexo: masculino o femenino.

4. Grupo de edad del paciente: de acuerdo a los utilizados en el SGSSS al momento del estudio(26);

5. Diagnóstico presuntivo al momento de realizar el Triaje: utilizando la Clasificación Internacional de Enfermedades (CIE-X) definida por la OMS, agrupada en listas especiales de 298 causas con el propósito de facilitar los análisis(27).

6. Clasificación del Triaje: de acuerdo a la clasificación de cuatro categorías propuesta por la cámara de salud de la ANDI, aceptada y utilizada en Colombia al momento de realizar esta investigación(28,29).

Con el objeto de garantizar que: la información estuviera completa, y que los RT no estuvieran repetidos en cuanto que un mismo usuario apareciese más de una vez en idéntica fecha y hora, fueron sometidos a filtros de calidad consistentes en: revisar que el registro en la base de datos contenga la información requerida por las variables solicitadas, que no hubiera duplicidad en estos, verificar que los formatos de la base de datos estuvieran de acuerdo a la información solicitada, por ejemplo que la fechas y los códigos del CIE-X estuvieran adecuadamente digitados, de tal forma que se pudiera relacionar esta información con la de la base de datos de códigos de CIE.

De acuerdo con lo reportado por las tres IPS, fueron recibidos 214.945 registros, de los cuales superaron el filtro de calidad 183.908, que correspondieron al $88 \%$ del total inicial. Los registros utilizados por IPS se distribuyeron así: la IPS1 aportó el 33,2\%, la IPS2 el 46,8\% y la IPS3 el 20\%.

El análisis estuvo integrado por dos componentes: el primero de tipo estadístico descriptivo y el segundo, de tipo analítico bivariado. El análisis estadístico descriptivo consistió en calcular proporciones y frecuencias relativas agrupadas en: característica de los usuarios, impresiones diagnósticas y distribución cronológica de la demanda; con el propósito de identificar patrones de uso del servicio de urgencias.

El analítico bivariado buscó establecer a priori la hipótesis de que existe relación de asociación entre la Clasificación del Triaje y las siguientes variables categóricas:

1. Condición de afiliación.

2. Segmento del día.

3. Día de la semana y segmento del día.

4. Segmento del día y condición de afiliación.

5. Edad y segmento del día.

Las hipótesis se plantearon de la siguiente manera:

$\mathbf{H}_{\mathbf{0}}$ : las variables son independientes

$\mathbf{H}_{\mathbf{1}}$ : las variables son dependientes

Teniendo en cuenta lo expuesto por Bewick et al., en su artículo sobre los test de asociación para variables cualitativas, la prueba seleccionada para establecer relaciones entre las variables 
categóricas fue la de Chi cuadrado, debido a que se estaba frente a un problema de comparación entre grupos independientes (clasificación por Triaje, grupos de edad, segmento del día en el que fue atendido, día de la semana en el que se requirió la atención) y variables categóricas(30). El análisis de datos se realizó utilizando el software SPSSS versión $20{ }^{\circledR}$ y Excel $2013{ }^{\circledR}$.

\section{Consideraciones éticas}

El presente estudio tuvo en cuenta las normas científicas, técnicas y administrativas para la investigación en salud en Colombia contenidas en la Resolución 8430 de octubre 4 de 1993 y los principios éticos contemplados en la Declaración de Helsinki(31,32).

El estudio contó con la aprobación del Comité Ética y Bioética de las IPS participantes y del comité de Ética de la Universidad Jorge Tadeo Lozano según Acta número 15 del 2 de Mayo de 2013. Se excluyó una cuarta IPS cuyo comité de ética no aprobó el suministro de la información requerida. En adición, para el caso de los RT de los usuarios, se omitió el campo de los nombres y el número de identificación se remplazó con un código de localización que permitió identificar el registro de manera anónima. Con el propósito de preservar la confidencialidad de las instituciones participantes, les fue asignado un código aleatorio a cada una de ellas para no emplear su nombre.

\section{Resultados}

A continuación, se presentan los resultados en dos acápites, el primero que da cuenta del análisis estadístico descriptivo y el segundo, que presenta los derivados del análisis bivariado.

\section{Análisis estadístico descriptivo}

Los resultados del análisis descriptivo muestran que el $54 \%$ de los registros correspondieron a usuarios de sexo femenino. Por grupo de edad, según grupos del SGSSSS, predominó el grupo de 15 a 44 años con el $57 \%$ de los registros, en segundo lugar, el grupo de 45 a 59 años con un $12 \%$ de los registros y en tercer lugar el grupo de edad de 1 a 4 años con un 10\%. Los adultos mayores y las personas entre cinco a 14 años representaron respectivamente cada uno el 9\% de los registros. Con respecto a la condición de afiliación al régimen contributivo del SGSSS, predominaron los cotizantes, seguidos por los beneficiarios y registros sin condición de afiliación (Figura 1).

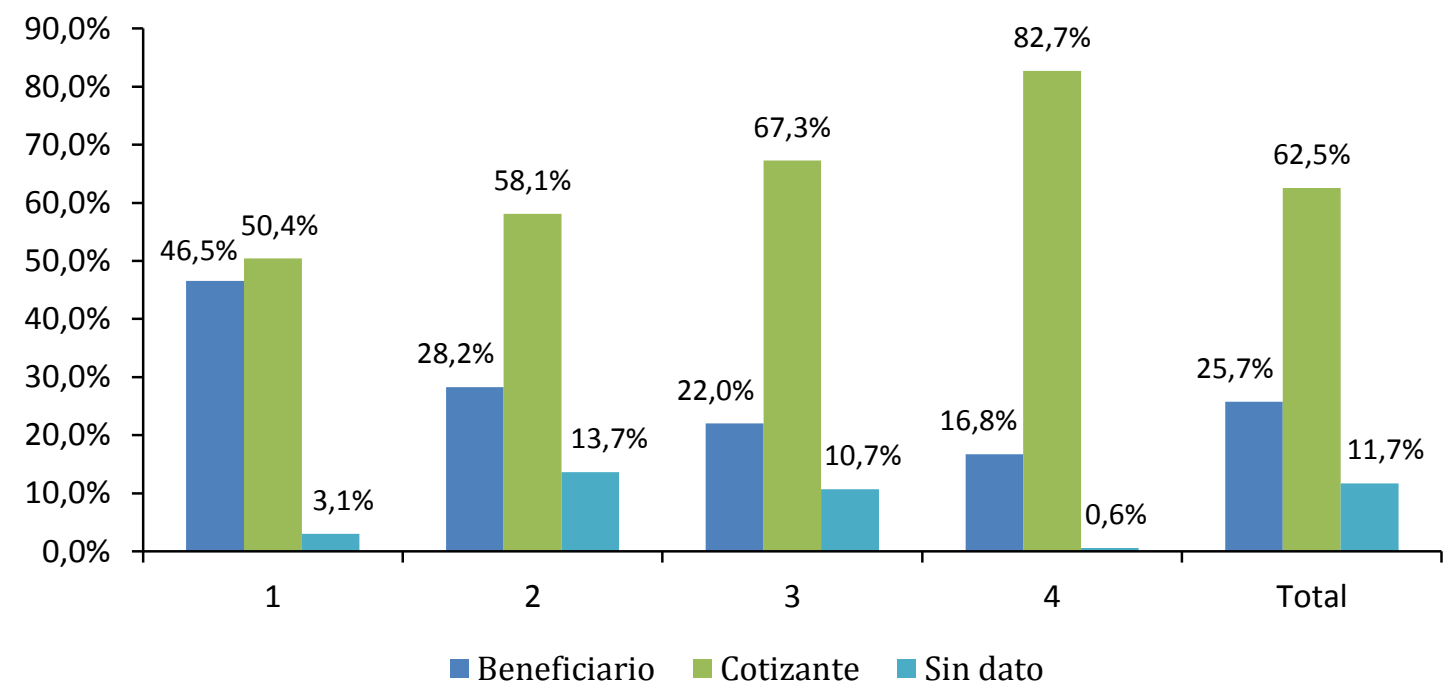

Figura 1. Participación por grupo y total según condición de afiliación de registros de Triaje de urgencias de tres IPS de Bogotá D. C. (1 de octubre 2012 - 31 de marzo 2013) 
Por clasificación el mayor porcentaje de pacientes se concentró en el Triaje 2 con el $64,3 \%$, seguido del Triaje 3 con $26,8 \%$, mientras que el Triaje 4 participó con el $8.3 \%$ y el Triaje 1 participó con el $0.6 \%$. Al analizar el uso por condición de afiliación al sistema, se observa que a medida que incrementa la clasificación del
Triaje aumenta la participación de cotizantes en relación con los beneficiarios (Figura 1).

Se encontró que el día lunes fue el que tuvo mayor participación de registros de atención mientras que el día domingo tuvo la menor (Figura 2).

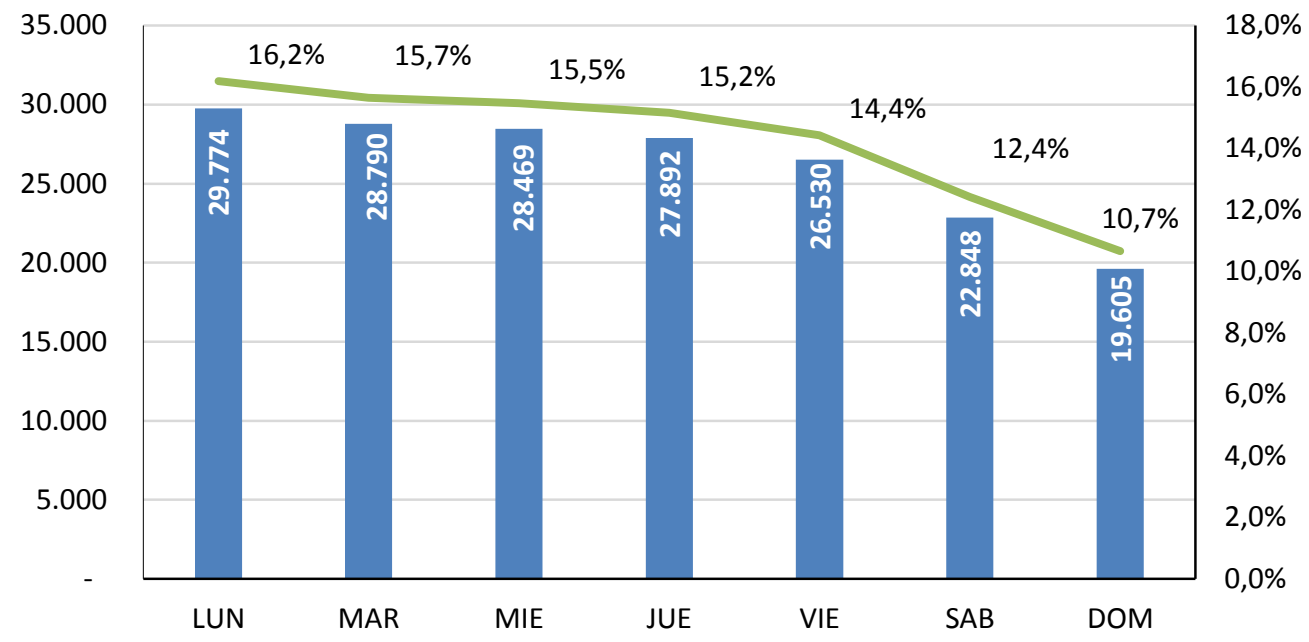

Figura 2. Distribución por día de la semana de registros de Triaje en urgencias de tres IPS de Bogotá DC

En relación con el segmento del día y la distribución de la atención, la mañana concentró el 36\% de los registros, la tarde el 34\%, la noche el $23 \%$ y la madrugada el $7 \%$. La mayor parte de los usuarios hicieron uso del servicio de urgencias entre las 7 de la mañana y las 8 de la noche con un pico en cuanto a la frecuencia del uso entre las 9 y 11 de la mañana (Figura 3).

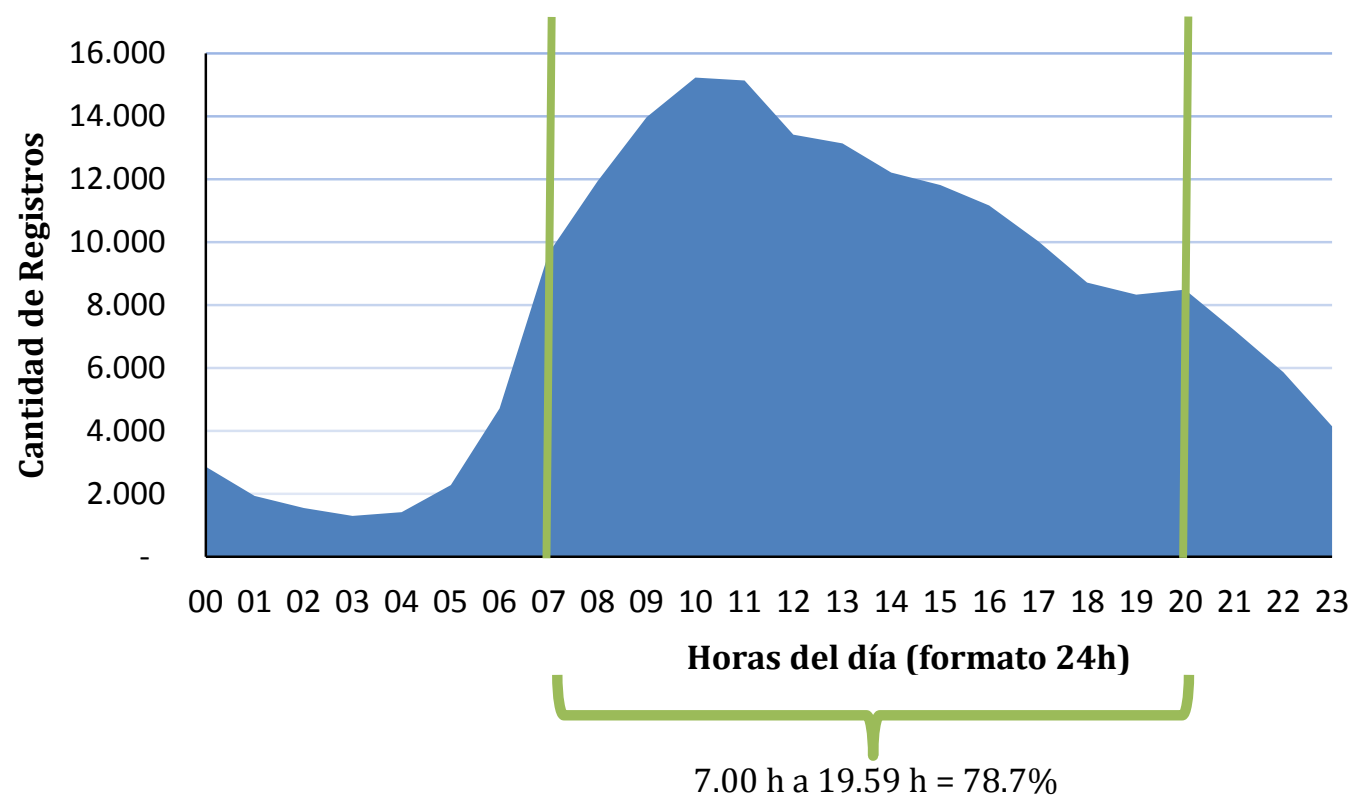

Figura 3. Distribución según hora del día de registros de Triaje en urgencias de tres IPS de Bogotá D. C. 
En la primera impresión diagnóstica se halló que para el Triaje I y II fue otros traumatismos, mientras que para el Triaje III y IV fue diarrea y gastroenteritis (Tabla 1).

Tabla 1. Cinco primeros grupos de diagnóstico según clasificación de Triaje en urgencias en tres IPS de Bogotá D.C.

(1 de octubre de 2012 - 31 de marzo de 2013)

\begin{tabular}{|c|c|c|}
\hline Triaje & Nombre_298 & $\%$ Total \\
\hline 1 & $\begin{array}{l}\text { Otros traumatismos de regiones especificadas, de regiones no especificadas y de múltiples regiones } \\
\text { del cuerpo }\end{array}$ & $34 \%$ \\
\hline 1 & Dolor abdominal y pélvico & $10 \%$ \\
\hline 1 & Diarrea y gastroenteritis de presunto origen infeccioso & $8 \%$ \\
\hline 1 & Otras infecciones agudas de las vías respiratorias superiores & $8 \%$ \\
\hline 1 & Bronquitis aguda y bronquiolitis aguda & $5 \%$ \\
\hline 2 & $\begin{array}{l}\text { Otros traumatismos de regiones especificadas, de regiones no especificadas y de múltiples regiones } \\
\text { del cuerpo }\end{array}$ & $26 \%$ \\
\hline 2 & Dolor abdominal y pélvico & $12 \%$ \\
\hline 2 & Diarrea y gastroenteritis de presunto origen infeccioso & $9 \%$ \\
\hline 2 & Otras infecciones agudas de las vías respiratorias superiores & $7 \%$ \\
\hline 2 & Faringitis aguda y amigdalitis aguda & $5 \%$ \\
\hline 3 & Diarrea y gastroenteritis de presunto origen infeccioso & $18 \%$ \\
\hline 3 & $\begin{array}{l}\text { Otros traumatismos de regiones especificadas, de regiones no especificadas y de múltiples regiones } \\
\text { del cuerpo }\end{array}$ & $9 \%$ \\
\hline 3 & Faringitis aguda y amigdalitis aguda & $9 \%$ \\
\hline 3 & Otras infecciones agudas de las vías respiratorias superiores & $9 \%$ \\
\hline 3 & Dolor abdominal y pélvico & $7 \%$ \\
\hline 4 & Diarrea y gastroenteritis de presunto origen infeccioso & $30 \%$ \\
\hline 4 & Otras infecciones agudas de las vías respiratorias superiores & $15 \%$ \\
\hline 4 & Faringitis aguda y amigdalitis aguda & $11 \%$ \\
\hline 4 & Otras dorsopatías & $9 \%$ \\
\hline 4 & Migraña y otros síndromes de cefalea & $4 \%$ \\
\hline
\end{tabular}

\section{Análisis bivariado}

Con respecto a la prueba de independencia de las variables, al realizar el test de Chi cuadrado $\left(X^{2}\right)$, se encontraron los siguientes resultados: existe dependencia entre condición de afiliación y el nivel de Triaje asignado $X^{2}=4884,774 \mathrm{p}<0,001$. Hay una asociación entre la clasificación del Triaje asignada y el segmento del día en el que se asiste al servicio de urgencias $X^{2}=4884,774$ $\mathrm{p}<0,001$. De igual forma hay una dependencia entre la clasificación del Triaje asignado, el día de la semana y segmento del día $X^{2}=427,8 \mathrm{p}<0,001$. También se encuentra que hay una dependencia estadísticamente significativa respecto al segmento del día y condición de afiliación al SGSS $X^{2}=4884,774 \mathrm{p}<0,001$. Finalmente se encuentra una asociación estadísticamente significativa entre la clasificación del Triaje, edad de la persona que consulta y segmento del día al que busca el servicio de urgencias $X^{2}=20439,314$ $\mathrm{p}<0,001$.

\section{Discusión y conclusiones}

El estudio evidencia que la mayor parte de los usuarios de los servicios de urgencias de las IPS incluidas, lo hicieron por condiciones que efectivamente se consideran urgentes de acuerdo a la clasificación empleada. Este hallazgo es consistente con lo reportado en la revisión adelantada por Oterino et al.(33), pero a su vez, es contrario a lo reportado en Cuba por Díaz y 
Guinart(34) y Pereda et al.(35), en los que la proporción de casos urgentes es similar a la de casos no urgentes; y en el contexto local, con el resultado obtenido en Medellín en 2007, donde fue mayor la cantidad de consultas consideradas no urgentes(23). Esto puede ser debido a criterios subjetivos tales como empatía entre quien clasifica y el paciente, el lenguaje en que se expresan los síntomas por parte del paciente, estados de ánimo de quien clasifica, el nivel de entrenamiento, entre otros; o también por el nivel de complejidad de las urgencias de las IPS estudiadas. En éste punto es importante acotar que dado el relativo reciente diseño del Sistema de Clasificación de Manchester, hasta ahora está siendo evaluado en cuanto a su precisión, en cómo afecta la evolución de los pacientes y el tiempo demandado para su aplicación; dependiendo de los resultados, este sistema de clasificación podría llegar a convertirse en una opción alternativa a la forma tradicional de clasificación del Triaje de origen francés(16).

La literatura presenta divergencias en cuanto al uso predominante del servicio de urgencias por parte del sexo femenino, algunos autores encontraron relación estadísticamente significativa entre el sexo femenino y el mayor uso de los servicios de urgencias $(24,34)$; otros encontraron esta misma relación sin establecer significancia estadística(33) y; por el contrario otras investigaciones presentan un mayor uso de los servicios de urgencias por parte del sexo masculino(36,37). Este resultado deberá ser analizado en futuros estudios, con el fin de identificar si se origina en condiciones culturales, al rol de las mujeres como trabajadores y responsables del hogar o por condiciones del sistema de salud.

Dentro de la revisión bibliográfica realizada, no se encontró algún estudio que analizara las dinámicas de uso entre quienes son trabajadores y quienes son los demás integrantes de su grupo familiar. El hecho de que la mayoría de los usuarios del servicio de urgencias sean cotizantes y que esta relación sea inversamente proporcional a la gravedad del paciente según clasificación del Triaje, puede indicar como lo expresan Tudela y Mòdol(37) a "la existencia de factores sociales extrínsecos a la prestación del servicio de urgencias", en el caso de estudio, aplicable a la dinámica entre la condición de afiliación al sistema de salud y el uso inadecuado del servicio de urgencias.

El hallazgo de que los principales usuarios de los servicios de urgencias son adultos jóvenes; discrepa un estudio de Bogotá en 2007 realizado por Paredes et al.(24); en el que se informa que son las poblaciones en los extremos de la vida (niños y adultos mayores), quienes más usan los servicios de urgencias, hallazgo que es ratificado en la literatura internacional(11,15,16,33,34,36). Esta discrepancia debería analizarse más a fondo para determinar si se debe a la existencia de incentivos ocultos, de orden social, económico o de condiciones del sistema de salud, para motivar el uso de los servicios de urgencias por parte de la población adulta joven y trabajadora.

El estudio estableció que es el día lunes es en el que los usuarios más asisten a los servicios de urgencias, reafirmando lo encontrado en Cuba por Díaz y Guinart(34) en su estudio del año 2007 y concordando con los hallazgos publicados por Paredes et al.(24) en Bogotá hace un lustro atrás; sin embargo, el estudio de Oterino et al., realizado en España no reporta significancia estadística con el mayor uso en dicho día(33) y por el contrario, Carbonell et al., determinaron un mayor uso los días martes o miércoles(36). Los datos hallados en esta investigación, así como los reportados en la literatura, permiten inferir que en los días del fin de semana se hace menor uso de los servicios de urgencias; esto deberá estudiarse para establecer si está o no asociado a factores culturales que inducen a aplazar la asistencia al servicio para los días hábiles de la semana(24,33,37). Con respecto a los resultados del análisis descriptivo es importante resaltar cómo la mayoría de las consultas se producen el lunes, día que comienza la semana laboral por lo que hay que tener en cuenta esto como una referencia para realizar actividades de intervención orientadas a disminuir la congestión de urgencia ese día de la semana.

Respecto a las características del uso de los servicios a lo largo del día, se encontró que la 
mayor demanda se da en el rango comprendido entre las 7:00 am y las 8:00 pm, con un pico máximo ubicado entre las 9:00 am y las 11:00 am. La literatura reporta hallazgos similares, con pequeñas divergencias en cuanto al momento de inicio y final reportados por Díaz y Guinart(34), o sobre el periodo de mayor demanda del servicio durante el día, según el estudio realizado en Bogotá por Paredes et al.(24). Este comportamiento debe estudiarse para identificar los factores de orden social, económico o de los servicios de salud que lo pueden originar.

Las causas de asistencia varían de acuerdo con el nivel del Triaje en el que fue clasificado el usuario. Se observa una preponderancia del trauma en los Triaje I y II, mientras que la diarrea es la principal causa de asistencia en los Triaje III y IV; esto resulta coincidente con lo hallado en investigaciones adelantadas en Colombia como el estudio realizado por Paredes et al.(24); pero, al comparar estos resultados con la literatura internacional, se encuentra que para las causas no urgentes (Triaje III y IV), los hallazgos son coincidentes; sin embargo, para las causas urgentes (Triaje I y II), los hallazgos no son coincidentes con la mayoría de lo reportado en la literatura internacional sobre este tema $(3,16,33)$; exceptuando lo reportado por Lee et al.(38), quienes encontraron que el trauma es una de las principales causas de asistencia a urgencias en Hong Kong.

Ahora bien, el hecho de que el trauma sea la principal causa de clasificación en Triaje I y II, tanto en el presente estudio como en el realizado en Bogotá por Paredes et al.(24), se sugiere sea objeto de investigaciones posteriores que identifiquen si este patrón es propio solo de Bogotá o también del país y, que también, puedan establecer si el origen corresponde a accidentalidad vial, laboral, violencia o a la combinación de estos factores.

Por su parte, el análisis de los resultados de los cruces de variables mediante tablas de contingencia muestra asociaciones con significancia estadística entre la condición de afiliación-nivel del Triaje, entre horaclasificación del Triaje, Triaje-día-segmento del día, condición de afiliación-día-clasificación del Triaje, clasificación del Triaje-edad-segmento del día. La existencia de estas asociaciones hace sospechar de la posible presencia de factores sociales externos a los servicios de urgencias de tipo social y cultural, que pueden estimular la conducta de asistir por parte de los individuos en días laborales y en horas hábiles a los servicios de urgencias por parte de trabajadores, mujeres y adultos jóvenes ${ }^{(11)}$. Se puede concluir que, con base en los datos recolectados para esta investigación, el uso de los servicios de urgencias está influenciado por la relación entre las características: demográficas, de condición de afiliación al sistema de salud y los patrones de uso del servicio de urgencias. Por tanto, se recomienda a los responsables de la gestión de estos servicios que contemplen este tipo de variables dentro de la planeación de los mismos.

Este estudio aporta otras variables al análisis de la problemática de los servicios de urgencias en cuanto a las dificultades de la correcta clasificación de los pacientes de acuerdo al Triaje como lo relaciona el estudio de Palma et al., en 2014 realizado en Italia(39). De igual forma en cuanto a la oportunidad de la prestación del servicio realizado por Hosseininejad et al., en Irán ${ }^{(40)}$ que centran su análisis en la atención de urgencias, mientras que el presente análisis se centra en un momento previo como es la clasificación de Triaje que resulta fundamental para permitir una atención oportuna.

\section{Limitaciones}

El hecho de que el estudio abarcó el análisis de un período de tiempo corto y en tres IPS de la ciudad de Bogotá, limita el alcance de los hallazgos y de las conclusiones, las cuales deberán ser corroboradas o refutadas mediante un análisis más amplio en tiempo y en IPS participantes.

\section{Recomendaciones}

De acuerdo a los resultados se observa una asociación entre la clasificación de Triaje con una serie de variables sociodemográficas y unos patrones horarios en el uso del servicio. Esto implica que los encargados de la toma de decisiones del servicio deben tener en cuenta 
estos aspectos en orden de aumentar la capacidad de atención en dichos horarios, así como profundizar en las causas que llevan a que se mejore el acceso al servicio de salud de afiliados que en razón a su trabajo no tienen la disponibilidad de tiempo de asistir el médico en su horario laboral diurno.

\section{Agradecimientos}

A las instituciones que facilitaron el acceso a los datos del registro de Triaje, a Fernando Betancourt por su orientación en el abordaje del problema, a Edgar Dorado Palacios por su apoyo en el procesamiento de la información y a los colaboradores Gino Montenegro, Paola Montoya y Carolina Bautista.

\section{Financiación}

Artículo de investigación resultado del proyecto de consultoría "Estudio piloto para conocer la pertinencia en el uso de los servicios de urgencias en Bogotá D.C. en relación con la capacidad instalada de dichos servicios y su relación con las lógicas de los usuarios asociadas al uso de los servicios de urgencias, su grado de aceptación o rechazo", adelantado por la Universidad Jorge Tadeo Lozano y financiado por la Asociación Colombiana de Empresas de Medicina IntegralACEMI y por la Cámara Sectorial de Salud de la Asociación Nacional de Industriales - ANDI, durante los meses de Mayo a Julio de 2013. Resultados preliminares de éste trabajo han sido presentados como Estudio Piloto Uso y capacidad instalada de los servicios de urgencias, en el Congreso Modelos Innovadores en Salud, Bogotá, D.C Colombia, mayo de 2013 y, en el XXXII Simposio Lasallista de Optometría, Bogotá D.C, octubre 2 de 2014. El reporte de resultados de investigación presentado en este artículo tiene el aval de las instituciones financiadoras.

\section{Conflicto de intereses}

Ninguno declarado por los autores.

\section{Referencias}

1. Durand AC, Palazzolo S, Tanti-Hardouin N, Gerbeaux P, Sambuc R, Gentile S. Nonurgent patients in emergency departments: Rational or irresponsible consumers? Perceptions of professionals and patients. BMC Res Notes [Internet]. 2012;5(525):1-9. Available from: http://www.biomedcentral.com/1756-0500/5/525

2. González G, Lía Valencia M, Armando Agudelo N, Acevedo L, Cristina Vallejo I. Morbilidad sentida de las urgencias médicas y la utilización de los servicios de salud en Medellín, Colombia, 2005-2006. Biomédica. 2007;27:180-9.

3. López Pons P. Calidad, Satisfacción y Patologías en un servicio de urgencias. Madrid: Universidad Autónoma de Madrid; 2013.

4. Vargas-Lorenzo I, Vázquez-Navarrete ML, MogollónPérez AS. Acceso a la atención en salud en Colombia. Rev Salud Pública [Internet]. 2010;12(5):701-12. Available from: https://revistas.unal.edu.co/index.php/revsaludpublic a/article/view/33310

5. Bedoya-Marrugo EA. Atención de urgencias en Cartagena, Colombia. Nova, [S.l] [Internet]. 2017;15(27):91-101. Available from: http://hemeroteca.unad.edu.co/index.php/nova/articl e/view/1961

6. Ramírez Estrada MC, Gil Ospina AA. Percepción de calidad en servicios de urgencias hospitalarias de II nivel, Risaralda, Colombia, 2013. Investig Andin [Internet]. 2016;18(32):1507-20. Available from: http://www.redalyc.org/pdf/2390/239047318006.pdf

7. Morales JC, Aranda-López-King JM, Cala-García SM. Propuesta de una solución tecnológica como alternativa a los problemas de saturación en las salas de urgencias de Bogotá, Colombia. Rev Cuba Inf en Ciencias la Salud [Internet]. 2017;28(2):1-10. Available from: http://scielo.sld.cu

8. Juárez-Flores DL, Alcántara ÁC, Castro PM. Urgencias médicas atendidas en el área de choque del hospital (Medical emergencies handled in the shock area of the hospital). Rev Mex Pediatría [Internet]. 2012;79(3):124-8. Available from: http://www.medigraphic.com/pdfs/pediat/sp2012/sp123c.pdf

9. Serrano-Benavente RA. Sistema de Triaje en Urgencias Generales. Universidad Internacional de Andalucía; 2013.

10. Ruiz-Rubiano DR. Diseño y evaluación de intervenciones en Organizaciones empresariales desde la dinámica de sistemas Triage. Bogotá: Universidad del Rosario; 2014.

11. Molano-Gutiérrez FR, Escobar-Grisales CM, GarcíaVargas MA, Salazar-Molina PA, Mejía-Venegas LC, Jiménez Barbosa WG. Motivos de uso de los servicios de urgencias. Acta Odontológica Colomb Enero-Junio [Internet]. 2016;6(1):123-36. Available from: https://revistas.unal.edu.co/index.php/actaodontocol/ article/view/58854

12. Hernández Sánchez H. Empleo del triaje. Rev Cuba Med Mil. 2013;42(3):472-83.

13. Cubero-Alpízar C, Medina-Arias K. Los procesos de clasificación de usuarios y su impacto en la atención en las salas de urgencias a nivel hospitalario. Enfermería Actual en Costa Rica [Internet]. 2013;(24):1-12. Available from: http://www.redalyc.org/articulo.oa?id=44826081009

14. Moreira DDA, Beatriz H, Tibães B, Rocha RC. O Sistema 
De Triagem De Manchester Na Atenção Primária À Saúde: Ambiguidades E Desafios Relacionados Ao Acesso Manchester Triage System in Primary Health Care : Ambiguities and Challenges Related To Access El Sistema De Triage De Manchester En La Atenció. Texto Context Enferm [Internet]. 2017;26(2):1-8. Available from: http://dx.doi.org/10.1590/010407072017005970015

15. Guedes HM, Araújo FA, Júnior DP, Martins JCA, Chianca TCM. Outcome assessment of patients classified through the Manchester Triage System in emergency units in Brazil and Portugal. Investig y Educ en Enferm [Internet]. 2017;35(2):174-81. Available from: http://docplayer.net/49100933-Outcome-assessmentof-patients-classified-through-the-manchester-triagesystem-in-emergency-units-in-brazil-and-portugal.html

16. Anziliero F, Dal Soler BE, Da Silva BA, Tanccini T, Gomez Beghetto M. Manchester System: time spent on risk classification and priority of care at an emergency medical service. Rev Gaúcha Enferm [Internet]. 2016;37(4):1-6. Available from: www.scielo.br/rgenf\%5Cnwww.scielo.br/rgenf\%5Cnw ww.scielo.br/rgenf\%5Cnwww.scielo.br/rgenf

17. Vargas-Zea N, Castro H, Rodríguez-Páez F, Téllez D, Salazar-Arias R. Colombian Health System on its Way to Improve Allocation Efficiency-Transition from a Health Sector Reform to the Settlement of an HTA Agency. Value Heal Reg Issues [Internet]. 2012;(1):218-22. Available

from: https://www.ispor.org/ValueInHealth/ShowValueInHe alth.aspx?issue=A6CA8C55-657C-4C15-86D34E52AB5FE0E3

18. Arroyave Zuluaga ID. La organización de la salud en Colombia [Internet]. Primera. Hospital Universitario San Vicente de Paúl, editor. Medellín: Periódico El Pulso; 2009. 585 p. Available from: www.periodicoelpulso.com.

19. Colombia: Ministerio de Salud y Protección Social. Sistema Integral de Información de la Protección Social. SISPRO [Internet]. Bogotá: MinSalud; 2015 [cited 2015 Sep 16]. Available from: http://www.sispro.gov.co/Pages/ContruyaSuConsulta/ Aseguramiento.aspx

20. Colombia: Ministerio de Salud y Protección Social. Sistema Integral de Información de la Protección Social. SISPRO [Internet]. Bogotá: MinSalud; 2017 [cited 2005 Jul 20]. Available from: http://www.sispro.gov.co/Pages/Contruya $\quad \mathrm{Su}$ Consulta/Aseguramiento.aspx

21. ACEMI. Cifras e indicadores del Sistema de Salud, 2013 [Internet]. 1st ed. Cifras e indicadores del sistema de salud. Bogotá: Acemi; 2013. 1-170 p. Available from: https://acemi.org.co/images/publicaciones/document os_de_interes/documentos-interes/salude_en_cifras2013.pdf

22. ACEMI. Cifras e indicadores del sistema de salud, 2017 [Internet]. Bogotá: Acemi; 2017. 432 p. Available from: https://issuu.com/acemi/docs/informe_cifras_2017

23. Valencia-Sierra ML, González-Echeverri G, AgudeloVanegas NA, Acevedo-Arenas L, Vallejo-Zapata IC. Acceso a los Servicios de Urgencias en Medellín, 2006.
Rev Salud Pública [Internet]. 2007;9(4):529-40. Available from: http://www.scielo.org.co/scielo.php?pid=S012400642007000400005\&script=sci_arttext

24. Paredes Zapata GD, Paz Velilla AI, González Echeverri G, Valencia Sierra ML, Agudelo Vanegas NA, García CM. Análisis del comportamiento de las consultas de urgencias RIPS 2004. Investig en Segur Soc y salud. 2007;1(9):140-55.

25. Colombia: Presidencia de la República. Decreto 056 de 1975. Diario Oficial No 34260 [Internet]. 1975 Feb 19; Available from: https://docs.supersalud.gov.co/PortalWeb/Juridica/De cretos/D0056_75.pdf

26. Colombia: Consejo Nacional de Seguridad Social en Salud. Acuerdo 351 de 2006 [Internet]. Bogotá: Consejo Nacional de Seguridad Social en Salud; 2006. Available from:

https://www.icbf.gov.co/cargues/avance/docs/acuerd o_cnsss_0351_2006.htm

27. Organización Panamericana de la Salud. Clasificación estadística internacional de enfermedades y problemas relacionados con la salud. Primera. Washington: OPS; 1995.

28. ANDI - Proyecto La llave. Clasificación para la priorización de la atención de urgencias [Internet]. Bogota: ANDI; 2015 [cited 2015 Dec 11]. Available from:

http://www.andi.com.co/cssa/Documents/Triage 20Clasificación para la priorización de la atención de urgencias.pdf

29. ANDI - Proyecto La llave. Tabla de clasificación para la priorización de la atención de urgencias [Internet]. Bogotá: ANDI. 2015 [cited 2015 Dec 11]. Available from:

http://www.andi.com.co/cssa/Documents/Triage Tabla de clasificación para la priorización de la atención de urgencias .pdf

30. Bewick V, Cheek L, Ball J. Statistics review 8: Qualitative data - Tests of association. Crit Care [Internet]. 2004;8(1):46-53. Available from: https://www.ncbi.nlm.nih.gov/pmc/articles/PMC4200 70/pdf/cc2428.pdf

31. Colombia: Ministerio de Salud. Resolución 8430 [Internet]. Bogotá: Ministerio de Salud; 1993. Available from:

https://www.minsalud.gov.co/sites/rid/Lists/Bibliotec aDigital/RIDE/DE/DIJ/RESOLUCION-8430-DE1993.PDF

32. Asociación Médica Mundial. Declaración de Helsinki de la AMM - Principios éticos para las investigaciones médicas en seres humanos [Internet]. Políticas Actuales. Helsinki: WMA; 2017 [cited 2017 Sep 25]. Available from: https://www.wma.net/es/policiespost/declaracion-de-helsinki-de-la-amm-principioseticos-para-las-investigaciones-medicas-en-sereshumanos/

33. Oterino D, Peiró S, Librero J, Ridao M, Bernal-Delgado E. Utilización inadecuada de un servicio de urgencias hospitalario. Una evaluación con criterios explícitos. Gac Sanit [Internet]. 2010;13(5):6-12. Available from: 
https://ac.els-cdn.com/S0213911199713877/1-s2.0S0213911199713877-main.pdf?_tid=673ac4a6-e2e8485a-809f-

01f110d2eda1\&acdnat $=1524696098 \_0$ cb7c3fb16f37ba 0d78ab28b6533a0b8

34. Díaz Novás J, Guinart Zayas N. Las urgencias en la atención primaria. Utilización del servicio. Rev Cuba Med Gen Integr [Internet]. 2007;23(4):1-8. Available from: http://scielo.sld.cu/pdf/mgi/v23n4/mgi02407.pdf

35. Pereda-Rodríguez J, Díaz-Sánchez I, Pereda-Rodríguez $\mathrm{R}$, Sosa-Acosta Á. Filtro sanitario en las urgencias médicas. Un problema a reajustar. Rev Cubana Med [Internet]. 2001;40(3):181-8. Available from: http://scielo.sld.cu/scielo.php?script=sci_arttext\&pid= S0034-75232001000300005\&lng=es.

36. Carbonell-Torregrosa M, Girbés-Borrás J, CalduchBroseta J. Determinantes del tiempo de espera en urgencias hospitalarias y su relación con la satisfacción del usuario. Emergencias [Internet]. 2006;18:30-5. Available from: http://emergencias.portalsemes.org/descargar/deter minantes-del-tiempo-de-espera-en-urgenciashospitalarias-y-su-relacion-con-la-satisfaccion-delusuario/force_download/

37. Tudela P, Mòdol JM. La saturación en los servicios de urgencias hospitalarios. Emergencias [Internet]. 2015;27:113-20. Available from: https://dialnet.unirioja.es/servlet/articulo?codigo $=538$ 8440

38. Lee A, Lau FL, Hazelett CB, Kam CW, Wong P, Wong TW, et al. Morbidity patterns of non-urgent patients attending accident and emergency departments in Hong Kong: cross-sectional study. Hong Kong Med J [Internet]. 2001;7(2):131-8. Available from: https://www.ncbi.nlm.nih.gov/pubmed/11514746

39. Palma E, Antonaci D, Colì A, Cicolini G. Analysis of Emergency Medical Services Triage and Dispatch Errors by Registered Nurses in Italy. J Emerg Nurs [Internet]. 2014;40(5):476-83. Available from: https://www.sciencedirect.com/science/article/pii/S0 099176714000828?via\%253Dihub

40. Hosseininejad SM, Aminiahidashti H, Pashaei SM, Khatir IG, Montazer SH, Bozorgi F, et al. Determinants of Prolonged Length of Stay in the Emer- gency Department; a Cross-sectional Study. Emergency [Internet]. 2017;5(1):e53. Available from: https://www.ncbi.nlm.nih.gov/pmc/articles/PMC5325 925/pdf/emerg-5-e53.pdf 